\section{¿Por qué la equidad en salud? ${ }^{1}$}

\author{
Amartya Sen ${ }^{2}$
}

Palabras clave: equidad, igualdad, justicia social.

\footnotetext{
1 Texto del discurso leído en la IIIConferencia Internacional sobre Economía de la Salud. York, Reino Unido, 23 de julio de 2001. Traducción publicada con el permiso del autor.

2 Premio Nobel de Economía, 1998.
}

"El mundo ... no es una fonda, sino un hospital", dijo Sir Thomas Browne hace más de tres siglos y medio, en 1643. Se trata de una interpretación del mundo desalentadora, si no totalmente sorprendente, hecha por el distinguido autor de Religio Medici y Pseudodoxia Epidemica. Pero es posible que Browne no estuviera completamente equivocado; incluso hoy, y no solo en la Inglaterra de Browne del siglo XVII, la enfermedad, de un tipo $u$ otro, tiene una importante presencia en la vida de muchísimas personas. De hecho, hasta puede que Browne haya sido algo optimista en su analogía con un hospital, pues hoy día muchas de las personas más enfermas no reciben ningún tratamiento para sus dolencias ni usan medios preventivos eficaces.

La enfermedad y la salud deben tener un lugar destacado en cualquier discusión sobre la equidad y la justicia social. Tomando como punto de partida esta ubicuidad de la salud como consideración social, empiezo por advertir que la equidad en salud no puede dejar de ser una característica central de la justicia de los acuerdos sociales en general. El alcance de la equidad en el campo de la salud es inmenso. Pero hay en esta relación otra característica a la que también debemos prestar atención. La equidad en salud no concierne únicamente a la salud, vista aisladamente, sino que debe abordarse desde el ámbito más amplio de la imparcialidad y la justicia de los acuerdos sociales, incluida la distribución económica, y prestando la debida atención al papel de la salud en la vida y la libertad humanas. Ciertamente, la equidad en salud no trata solo de la distribución de la salud, por no hablar del campo todavía más limitado de la distribución de la asistencia sanitaria. En efecto, la equidad en salud tiene un alcance y una relevancia enormemente amplios.

En esta charla consideraré tres asuntos. Primero empezaré por analizar la naturaleza e importancia de la equidad en salud. Después pasaré a identificar y analizar en profundidad las diferentes justificaciones que se han dado para reivindicar que la equidad en salud no es el asunto político en el que nos deberíamos centrar, y espero ser capaz de demostrar que los motivos de este escepticismo no se sostienen ante un análisis minucioso. Por último, en el tercer apartado analizaré algunas cuestiones difíciles que hay que plantearse para poder comprender de forma adecuada los requisitos de la equidad en salud. En este contexto resulta particularmente importante entender la equidad en salud como una disciplina muy amplia que tiene que acomodarse a consideraciones muy diversas y dispares. 


\section{EQUIDAD EN SALUD Y JUSTICIA SOCIAL}

En un trabajo anterior, publicado hace unos 10 años (1), traté de sostener que en el mundo contemporáneo ninguna teoría de la justicia podría tener verdadera credibilidad si no valorara la igualdad en algún espacio, en un espacio que esa teoría considerara importante. Un defensor del igualitarismo de ingresos, un paladín de la democracia, un liberal y un conservador defensor del derecho a la propiedad pueden tener distintas prioridades, pero todos quieren igualdad en algo que consideran valioso, de hecho fundamental, en su respectiva filosofía política. El defensor del igualitarismo de ingresos valorará la distribución igualitaria de los ingresos; el demócrata comprometido insistirá en la igualdad de derechos políticos para todos; el liberal decidido demandará igualdad de libertades, y el conservador defensor del derecho de propiedad insistirá en que todos tienen el mismo derecho a usar las propiedades que tengan. Todos ellos valoran mucho, y no de forma accidental, la igualdad en relación con alguna variable que ocupa una posición central en sus respectivas teorías de la justicia. En efecto, hasta un enfoque agregativo, como el del utilitarismo benthamita, implica una relación con la igualdad, en la medida en que todos deberían ser tratados del mismo modo al llegar a los agregados simples (como el total de utilidad).

De hecho, como idea abstracta, la igualdad no tiene mucha fuerza, y el trabajo real comienza con la especificación de qué es lo que hay que igualar. El paso fundamental consiste, pues, en especificar el ámbito en el que hay que buscar la igualdad y las reglas a seguir para definir lo equitativo en los aspectos tanto agregativo como distributivo. Las respuestas a preguntas como "¿igualdad de qué?" y "¿equidad de qué forma?" dependen del contenido de las respectivas teorías $(1,2)$.

Es aquí donde la salud adquiere un carácter crítico, haciendo que la equidad en salud sea fundamental para entender la justicia social. Sin embargo, es importante entender que la salud participa en el ámbito de la justicia social de varias formas distintas y que no todas proporcionan exactamente la misma lectura de acuerdos sociales particulares. En consecuencia, la equidad en salud es inevitablemente multidimensional. Si antes de emitir juicios inequívocos insistimos en buscar una congruencia de los diferentes aspectos de la equidad en salud, esta proporcionará a menudo una partición incompleta o un ordenamiento parcial. Esto no pone fin a la disciplina de la evaluación racional, ni siquiera de la maximización, que pueden enfrentarse a lo incompleto mediante la articulación reticente, pero va en contra de la esperanza que alientan algunos de que en toda comparación de estados sociales debe haber una jerarquía completa que sitúe todos los estados alternativos en un ordenamiento sencillo $(3,4))^{3}$ En efecto, aun cuando dos estados alternativos hayan sido finalmente clasificados de forma clara y decisiva, dicha clasificación puede basarse en la ponderación relativa, y quizás incluso en una reconciliación entre consideraciones divergentes, que retienen su relevancia separada y dispar incluso después de que se hayan evaluado sus pesos comparativos.

¿Cuáles son entonces las distintas consideraciones? Primero, la salud es una de las condiciones más importantes de la vida humana y un componente fundamental de las posibilidades humanas que tenemos motivos para valorar. Ninguna concepción de la justicia social que acepte la necesidad de una distribución equitativa y de una formación eficiente de las posibilidades humanas puede ignorar el papel de la salud en la vida humana y en las oportunidades de las personas para alcanzar una vida sana, sin enfermedades y sufrimientos evitables ni mortalidad prematura. La equidad en la realización y distribución de la salud queda así incorporada y formando parte integral de un concepto más amplio de la justicia.

Lo que es particularmente grave como injusticia es que algunos pueden no tener la oportunidad de alcanzar una buena salud debido a acuerdos sociales y no, digamos, a una decisión personal de no preocuparse particularmente por su salud. En este sentido, una enfermedad que no es prevenida ni tratada por motivos sociales (digamos por la pobreza o por la fuerza aplastante de una epidemia), y no por una elección personal (como el tabaquismo y otros comportamientos de riesgo de los adultos), tiene una repercusión particularmente negativa en la justicia social. Esto requiere una distinción adicional entre el logro de la salud y la posibilidad de alcanzar una buena salud, que se puede ejercer o no. Esta distinción es importante en algunos casos, pero en la mayoría de las situaciones el logro de la salud tiende a ser una buena guía de las posibilidades subyacentes, dado que tendemos a darle prioridad a la buena salud cuando realmente tenemos la oportunidad de elegir (en efecto, hasta el tabaquismo y otros comportamientos adictivos pueden ser vistos como una "falta de libertad" para oponerse al hábito, lo cual trae a colación el problema de la influencia psicológica sobre la posibilidad, tema que no abordaré aquí).

Es importante distinguir, por una parte el logro y la posibilidad, y por otra las facilidades sociales ofrecidas para dicho logro, como la atención sanitaria. Al contrario de lo que a veces se piensa, abogar

\footnotetext{
3 He analizado la necesidad de los ordenamientos incompletos y las articulaciones reticentes en dos obras anteriores (referencias 3 y 4 ).
} 
por la equidad en salud no puede consistir simplemente en demandas relacionadas con la distribución de la atención sanitaria en particular. Los factores que pueden contribuir a los logros y fracasos en el campo de la salud van mucho más allá de la atención sanitaria e incluyen muchas influencias muy distintas, desde las predisposiciones genéticas, los ingresos individuales, los hábitos alimentarios y los estilos de vida hasta el entorno epidemiológico y las condiciones de trabajo. ${ }^{4}$ Recientemente, Sir Michael Marmot y otros han sacado a relucir también los importantes efectos de la desigualdad social sobre la salud y la supervivencia (5-8). Para lograr una comprensión adecuada de los logros y posibilidades de salud tenemos que ir mucho más allá de la prestación y distribución de la atención sanitaria. La equidad en salud no se puede entender en términos de distribución de la atención sanitaria.

Segundo, en la medida en que los procesos y la justicia procesal tienen una importancia ineludible para la justicia social, tenemos que ir más allá de los logros de la salud y de la posibilidad de lograr la salud. Como alguien que ha realizado grandes esfuerzos para tratar de establecer la importancia de la perspectiva de posibilidad (incluidas las posibilidades de salud) con respecto a la justicia social, he de destacar también que la base informativa de la justicia no puede consistir únicamente en la posibilidad de información, dado que, además de los resultados y de la posibilidad de lograr los resultados valorados, también son importantes los procesos $(9,10)$. Por este motivo, las desigualdades, incluso en la atención sanitaria y no solo en el logro de la salud, también pueden ser importantes para la justicia social y la equidad en salud, dado que el aspecto procesal de la justicia y la equidad también requiere atención, sin ocupar necesariamente el centro del escenario.

Déjenme ilustrar esto con un ejemplo. Hay pruebas de que, en gran medida por motivos biológicos, las mujeres tienden a tener mayores probabilidades de supervivencia y menor incidencia de algunas enfermedades a lo largo de la vida; de hecho, hasta los fetos del sexo femenino tienen menor probabilidad de ser abortados espontáneamente. En efecto, esta es la principal razón de que en las sociedades con escasos o nulos sesgos de género en la atención sanitaria (como las de Europa Occidental y Norteamérica) predominen las mujeres, a pesar de que nazcan más niños que niñas en todo el mundo y de que se conciba una proporción todavía mayor de fetos masculinos. Si se juzga únicamente en tér-

\footnotetext{
4 La importancia de la distinción entre la salud y la asistencia sanitaria en la determinación de la política pública ha sido bien analizada, entre otros, por Jennifer Prah Ruger en Aristotelian justice and health policy: capability and incompletely theorized agreements. Disertación de tesis doctoral. Universidad de Harvard, 1998; a ser publicada por Clarendon Press.
}

minos de logros de salud y longevidad, esto representa una desigualdad relacionada con el género que solo está ausente en aquellas sociedades donde los sesgos antifemeninos de la atención sanitaria, y a veces también de la nutrición, hacen que la esperanza de vida de las mujeres no sea superior a la de los hombres. Pero sería moralmente inaceptable proponer que las mujeres deberían recibir peor atención sanitaria que los hombres con el fin de hacer desaparecer la desigualdad en el logro de la salud y la longevidad. ${ }^{5}$ La exigencia de la justicia de proceso requiere que ningún grupo - en este caso las mujeres - sea discriminado de esta forma; pero para defender esta conclusión tenemos que alejarnos, de una forma u otra, de una dependencia exclusiva de los logros de salud.

Tercero, la equidad en salud no puede preocuparse únicamente de la desigualdad en la salud o en la atención sanitaria, y debe tomar en consideración cómo se relaciona la salud con otras características a través de la asignación de recursos y de los acuerdos sociales. Una vez más, déjenme ilustrar esto con un ejemplo concreto. Supongamos que las personas A y B tienen exactamente las mismas predisposiciones desde el punto de vista de la salud, entre ellas la misma propensión a una enfermedad particularmente dolorosa. Pero A es muy rico y consigue curar o suprimir completamente su dolencia con algún tratamiento médico caro, mientras que $B$ es pobre y no puede pagarse ese tratamiento, por lo que sufre mucho con la enfermedad. Aquí hay una clara desigualdad en la salud. Además, si desde el punto de vista moral no aceptamos que el rico reciba un tratamiento privilegiado, se puede argumentar que también hay una violación de la equidad en salud. Concretamente, los recursos usados para curar al rico A podrían haberse usado para proporcionarle algún alivio a ambos o, en caso de indivisibilidad, para proporcionarle a ambos la misma probabilidad de obtener la curación a través de algún mecanismo probabilístico. No es difícil defender esta argumentación.

Ahora imagínense un cambio de política introducido por algún defensor de la igualdad sanitaria que le dé prioridad a la reducción de las desigualdades sanitarias y que le impida al rico A comprar una curación que el pobre B no pueda comprar. La vida del pobre B no se habrá visto afectada, pero ahora el rico A también vivirá con la dolorosa enfermedad y gastará su dinero, digamos que en viajes consoladores en un lujoso yate en mares exóticos. El cambio de política ha reducido, de hecho, la desigualdad en salud, pero ¿se puede decir que haya mejorado algo la equidad en salud? Para dejar clara la cuestión que

\footnotetext{
5 He analizado este asunto en Inequality reexamined (referencia número 1, capítulo 6).
} 
se está planteando, nótese que la pregunta no es si ahora la situación global es mejor (difícilmente se podría decir que sí); tampoco estoy preguntando si, considerándolo todo, es un arreglo justo. Una vez más, no lo es; parece más bien un cambio para peor de Pareto, dada la voluntad de A de gastar su dinero en comprar salud y no un yate. Concretamente, lo que pregunto es si hay más equidad en salud en este caso que en el anterior.

Yo diría que, aunque se haya reducido la desigualdad en el campo de la salud, no se ha mejorado la equidad haciendo que el rico A viaje por mares exóticos en su caro yate. Los recursos que ahora usa el rico A para viajar por alta mar en su yate se podrían haber empleado para curar al pobre $\mathrm{B}$ o al rico A, o para proporcionarle a cada uno de ellos algún alivio a sus penosas dolencias. La reducción de la desigualdad no ha mejorado la equidad en salud, dado que esta última necesita que consideremos, además, la posibilidad de conseguir acuerdos diferentes para la asignación de recursos, las instituciones o las políticas sociales. Evaluar la equidad en salud centrándose únicamente en la desigualdad es exactamente lo mismo que abordar el problema del hambre en el mundo comiendo menos y obviando el hecho de que se puede usar cualquier recurso natural para alimentar mejor a los hambrientos.

La violación de la equidad en salud no se puede juzgar exclusivamente en función de la desigualdad en salud. En efecto, se puede argumentar que algunos de los problemas políticos más importantes de la promoción de la salud son profundamente dependientes de la asignación global de recursos a la salud, y no únicamente de acuerdos distributivos de la atención sanitaria (por ejemplo, el "racionamiento" de la atención sanitaria y de otros determinantes de la salud), en los cuales parece estar concentrada en la actualidad gran parte de la literatura sobre la equidad en salud. Los recursos son fungibles y los acuerdos sociales pueden facilitar la salud de los pobres, a costa no solo de la atención sanitaria o de los logros de salud de otras personas, sino también a través de un acuerdo social diferente o de una modificación de la asignación de recursos. La magnitud de la desigualdad en salud no puede proporcionarnos información suficiente para evaluar la equidad en salud.

Evidentemente, esto no implica que la desigualdad en salud carezca de interés. Lo tiene por sí misma y es ciertamente una parte muy importante de nuestra forma de entender el concepto más amplio de equidad en salud. Por ejemplo, cuando hay grandes desigualdades en el logro de la salud que no se deben a precondiciones de salud irremediables, sino a la inexistencia de políticas económicas, reformas sociales o compromiso político, el hecho de la desigualdad en salud tiene una importancia considerable. No se pueden identificar las desigualdades en salud con la inequidad en salud, pero aquellas son indudablemente importantes para esta. No hay en esto contradicción alguna, una vez que consideremos la equidad en salud como un concepto multidimensional.

\section{ARGUMENTOS CONTRARIOS}

La reivindicación de la importancia de la equidad en salud puede ser objetada desde varios puntos de vista, con argumentos tanto empíricos como conceptuales. Estos argumentos contrarios han sido presentados de diferentes formas en discusiones tanto profesionales como populares. Es útil examinar las bases de estos diferentes argumentos y evaluar la importancia de la equidad en salud a la luz de estas críticas. Esto es lo que voy a hacer planteando algunas cuestiones escépticas como recurso dialógico.

1) ¿Son realmente importantes, en general, las demandas distributivas? Se podría argumentar que los requisitos distributivos en general, incluida la equidad, y no solo la equidad en salud, carecen de importancia ética como principio general. Los utilitaristas, por ejemplo, no están particularmente preocupados por la desigualdad en la utilidad, y en vez de ello se centran en la maximización de la suma total de utilidades, independiente de la distribución. Un rechazo fundamental de la preocupación por la desigualdad llevaría, entre otras cosas, a la reducción de la importancia de la equidad en salud.

Para responder a esto hay que considerar varios contraargumentos diferentes. Primero, como ha argumentado John Rawls al refutar las posturas del utilitarismo, la indiferencia a la distribución no toma con suficiente seriedad la distinción entre las personas (11). Si alguien sigue siendo infeliz o estando terriblemente enfermo, sus privaciones no se eliminan, remedian ni reducen simplemente haciendo que algún otro sea más feliz o más sano. Cada persona merece ser considerada como tal, y esto va en contra del enfoque indiferente a la distribución. El contraargumento rawlsiano es tan importante para las desigualdades en salud como en el bienestar o la utilidad.

Segundo, en el campo específico de la salud las posibilidades de que las personas puedan ser cada vez más sanas no son ilimitadas. En consecuencia, hasta el aspecto ingenieril de la estrategia de compensar la mala salud de unos con una salud cada vez mejor de otros tiene límites estrictos.

Tercero, aunque estuviéramos de alguna forma convencidos del enfoque indiferente a la distribución, seguiría habiendo alguna forma de consi- 
derar la equidad al tratar a todas las personas de la misma forma para llegar a logros agregados, como hace el utilitarismo. La maximización de la suma total independiente de la distribución no es tanto una negación de la equidad como una forma especial, y bastante limitada, de acomodar la equidad a las demandas de justicia social.

2) ¿Son las exigencias distributivas realmente importantes para el logro de la salud en particular? Se podría argumentar que la equidad puede ser importante en algunos campos, pero que cuando se trata de la falta de salud, cualquier reducción de la enfermedad en cualquier persona debe considerarse importante y recibir la misma prioridad, independientemente de cuál sea el nivel global de salud o de opulencia general de esa persona. La minimización de los AVAD (años de vida ajustados en función de la discapacidad), independientes de la distribución y muy usados en la actualidad, es un buen ejemplo de este enfoque (12-14).

Para responder a esta pregunta es útil empezar por reconocer explícitamente que cualquier mejoría de la salud de alguien es un buen principio para reconocer que ha habido alguna mejoría social. Pero esta necesidad de ser sensible a la salud de cualquiera no implica que se le conceda la misma importanncia al mejoramiento de la salud de cualquiera, independientemente de lo enfermo que esté. En efecto, como han señalado Sudhir Anand y Kara Hansen, la indiferencia a la distribución es una grave limitación del enfoque de los $\operatorname{AVAD}(15,16)$. El uso de la indiferencia a la distribución en el caso de los AVAD funciona de hecho con una cierta perversidad, dado que una persona discapacitada o con una enfermedad crónica, y por lo tanto con una desventaja general, con el ejercicio de minimización del AVAD también recibe menos atención médica para otras dolencias y esto tiene el efecto de añadir más desventaja relativa a alguien que ya está en desventaja. La crítica de Rawls a la indiferencia del utilitarismo a la distribución por no tomar suficientemente en serio la diferencia entre las personas se aplicaría aquí con fuerza redoblada.

En este contexto, es interesante notar que los fundadores (como Alan Williams y Tony Culyer) del enfoque AVAC (años de vida ajustados en función de la calidad), que posee alguna semejanza genérica con el enfoque AVAD, han sido partidarios de ajustar las cifras de los AVAC con consideraciones distributivas. ${ }^{6}$ En efecto, al exponer sus opiniones sobre lo que él llama el argumento del "turno equitativo"7, Alan Williams señala que "durante

\footnotetext{
6 Los exponentes de las estrategias AVAC y AVAD han discutido ampliamente sus diferencias en recientes debates entre York y Ginebra. Sin embargo, no trataré de esas diferencias en este ensayo.

7 "Fair innings" en inglés.
}

mucho tiempo" ha defendido "el punto de vista de que la mejor forma de integrar las consideraciones de eficiencia y equidad en la provisión de atención sanitaria sería darle a los AVAC ponderaciones ligadas a la equidad" (17-19). No hay motivos especiales para ser sensible a la equidad en general y no tener en cuenta la equidad en salud.

3) Teniendo las ideas amplias de equidad y justicia social en general, ipor qué necesitamos la noción más limitada de equidad en salud? Se puede argumentar que las consideraciones relacionadas con la equidad referida a la salud son absorbidas conceptualmente por alguna noción más amplia de la equidad, relacionada con, digamos, las utilidades o los derechos. Las consideraciones sobre la salud pueden figurar, por ejemplo, en el análisis global de la equidad social, pero desde esta perspectiva la equidad en salud carece de un estatus propio.

Esta crítica sería considerablemente convincente si la idea de equidad en salud estuviera destinada a desligarse de la de equidad y justicia en general. Pero, como ya hemos argumentado en este ensayo, la disciplina de la equidad en salud no está confinada a tratar solo de las desigualdades en salud. La equidad en salud puede formar parte integral del marco más amplio de la equidad en general, pero siempre habrá algunas consideraciones especiales relacionadas con la salud que forzosamente tendrán que integrarse en la evaluación de la justicia en general. Al hacer este ejercicio, la idea de equidad en salud plantea algunas cuestiones y perspectivas específicas que enriquecen la noción más abstracta de la equidad en general.

Hay que destacar que la salud es esencial para nuestro bienestar y que las libertades y posibilidades que somos capaces de ejercer dependen de nuestros logros en salud. Porque no podemos hacer muchas cosas si estamos discapacitados o incesantemente abrumados por la enfermedad y son muy pocas las que podemos hacer si no estamos vivos. Como dijo Andrew Marvel en su poema de 1681 titulado $A$ su tímida amante ("To His Coy Mistress"): La tumba es un lugar excelente y privado, / Pero nadie, creo, se abraza alli ("The grave's fine and private place,/ But none, I think, do there embrace"). La penalidad de la enfermedad puede no limitarse únicamente a la pérdida del bienestar, sino incluir también la falta de libertad para hacer lo que uno considere sus responsabilidades y compromisos (1, 20-24). La salud y la supervivencia son fundamentales para entender no solo la calidad de vida, sino también para que uno haga lo que quiere. Difícilmente se puede exagerar la importancia de la equidad en salud para la justicia social en general.

4) ¿La equidad en salud no es absorbida por las consideraciones de equidad en la distribución de los recursos, como los ingresos o lo que Rawls llama los "bie- 
nes primarios"? En esta línea de razonamiento se argumenta que, en principio, la equidad en salud puede tener alguna importancia, pero que es absorbida empíricamente por la atención que debemos prestar a la distribución de los recursos o "bienes primarios", dado que son estos recursos económicos y sociales los que, en última instancia, determinan el estado de salud de las personas.

Para responder a esto, podemos empezar por hacer notar que el estado de salud del que disfruta una persona está influenciado por diferentes consideraciones que nos llevan mucho más allá del papel de los factores sociales y económicos. Un enfoque adecuado de la política de salud debe tener en cuenta no solo las influencias de los factores sociales y económicos generales, sino también de una gran variedad de parámetros distintos, como las discapacidades personales, la propensión individual a la enfermedad, los riesgos epidemiológicos de cada región en particular, la influencia de las variaciones climáticas, etc. Una buena teoría de la equidad en salud tiene que darle a estos factores su valor dentro de la disciplina de la equidad en salud. En general, al hacer una política de salud es necesario distinguir entre la igualdad en los logros de salud (o las correspondientes posibilidades y libertades) y la igualdad en la distribución de lo que, en términos generales, se pueden llamar recursos sanitarios. Aunque estos últimos son importantes, he argumentado con consideraciones de proceso que es lo primero lo que ocupa una posición central en la equidad en general, y en la equidad en salud en particular.

\section{CONSIDERACIONES GENERALES Y PROPUESTAS PARTICULARES}

Por último, me centraré en las cuestiones y debates sobre los aspectos fundamentales del contenido de la equidad en salud. Dado que, como he tratado de argumentar, la equidad en salud tiene que ser vista como una disciplina amplia y no como un criterio estrecho y formulista, hay espacio para muchos enfoques diferentes en el ámbito de la idea básica de la equidad en salud. Pero la amplitud de la idea de equidad en salud necesita, por sí misma, alguna defensa. Los problemas y dificultades a la hora de adoptar una interpretación limitada de la equidad en salud no residen generalmente en la importancia de lo que afirma dicha interpretación (esto no suele estar en duda), sino en lo que niega. Es posible aceptar la importancia de una perspectiva sin que eso sea motivo suficiente para rechazar otras formas de enfocar la equidad en salud que también pudan ser importantes.

Consideremos la convincente idea de "turno equitativo" de Alan Williams $(17,25)$, que está rela- cionada con el enfoque de la equidad en salud desarrollado por Anthony Culyer y Adam Wagstaff, pero que lo amplia considerablemente $(18,19)$. Williams elabora la argumentación del turno equitativo con gran cuidado, señalando la ética subyacente a este enfoque: "la noción de un 'turno equitativo' se basa en la opinión de que todos tenemos derecho a un cierto grado de realización en el juego de la vida $\mathrm{y}$ de que cualquiera que no alcance ese grado ha sido maltratado por ella, mientras que cualquiera que lo supere no tendrá motivo para quejarse cuando se acabe su tiempo" (17 —página 319—). En el desarrollo de esta idea, Williams llega a la posición de que "si pensamos, como yo, que el turno equitativo debe definirse en términos de esperanza de vida al nacer ajustada por calidad y que deberíamos estar preparados para hacer algún sacrificio con el fin de reducir esa desigualdad, es muy factible calcular una serie de ponderaciones que representen el valor social diferencial del mejoramiento de los años de vida ajustados por calidad otorgados a diferentes clases de personas en nuestra situación actual." Mediante este procedimiento, Williams plasma hábilmente el importante problema de equidad relacionado con el hecho de que puede haber diferencias muy grandes entre las diferentes clases sociales en cuanto a la probabilidad de obtener un turno equitativo.

No hay duda de que este enfoque tiene mucho que elogiar y que, en particular, parece tratar de forma completa las desigualdades entre clases. No obstante, todavía se puede preguntar si esto es todo lo que se necesita plasmar al aplicar la idea de equidad en salud. Solo para plantear una cuestión elemental, déjenme volver a los menores riesgos de salud y a las mayores probabilidades de supervivencia de las mujeres en comparación con los hombres. Williams advierte este hecho y hace notar que "la diferencia de esperanza de vida al nacer entre los hombres y las mujeres del Reino Unido es todavía mayor que entre las clases sociales." Y a continuación señala que la diferencia entre géneros en cuanto a años de vida ajustados en función de la calidad es comparativamente menor que en cuanto a la esperanza de vida no ajustada (las mujeres parecen tener una vida más dura que los hombres), pero también hace notar que "mientras cerca del $80 \%$ de las mujeres sobreviven lo suficiente para disfrutar de un turno equitativo (que en este caso he considerado que son $60 \mathrm{AVAC}$ ), los hombres que logran esto son menos del $60 \%$."

Siguiendo esta línea de razonamiento, Williams dice que "Nosotros, los hombres, no estamos obteniendo un turno equitativo" (17 —página 327-). Las cuestiones más difíciles surgen después de haber reconocido esto. Entonces, ¿qué debemos hacer? $\mathrm{Si}$, como presupone el enfoque del turno 
equitativo, debe ser esto lo que guíe la asignación de recursos sanitarios, entonces tiene que haber desigualdad en la atención sanitaria a favor de los hombres, con el fin de restablecer el equilibrio. Pero ¿queremos realmente esa desigualdad en la atención? ¿No hay en la perspectiva de la igualdad de proceso algo que se resiste a esta conclusión y que va en contra de la provisión de una atención diferente en función del género de la persona cuando el hombre y la mujer sufren la misma dolencia?

El tema de la diferencia entre los géneros ilustra un problema más general, y es que las diferencias en la esperanza de vida ajustada por calidad no nos dan suficiente pie para ignorar las demandas de no discriminación en algunos campos fundamentales de la vida, como la necesidad de atención médica para las dolencias tratables. A veces las diferencias son muy sistemáticas, como ocurre con el género o la edad; en efecto, como señala Williams, "cualquiera que sea el grupo al que pertenezcamos, los supervivientes verán aumentar lentamente sus probabilidades de alcanzar un turno equitativo $\mathrm{y}$, a medida que mejoren sus perspectivas, las ponderaciones de equidad asociadas deberían disminuir" (17 - páginas 326-327-). El turno equitativo es un argumento persuasivo, pero no el único argumento persuasivo. Por ejemplo, no podemos negarnos a aceptar que el Rey Lear es una tragedia basándonos en el hecho de que, antes de que Sheakspeare empezara su historia, Lear había tenido una larga y buena vida, con muchos y excelentes años de vida ajustados en función de la calidad, que van más allá de un "turno equitativo".

Este problema no es particular de la propuesta de Alan Williams, sino que también se aplica a todos los enfoques que insisten en adoptar una visión unidimensional de la equidad en salud, en términos de logro de salud (o, en realidad, de posibilidad de lograr la salud). Por ejemplo, se aplica igualmente a la conclusión política a la que llegan Tony Culyer y Adam Wagstaff en su justamente celebrado artículo sobre "Equidad e igualdad en salud y en atención sanitaria" (18): que "la equidad en la atención sanitaria debería ... acarrear una distribución de la atención de tal forma que se llegue tan cerca como sea posible de una distribución igual de la salud." Pero, ¿en realidad debería? La concesión de preferencia, en el caso del género, a los pacientes varones y otras discriminaciones explícitas de este tipo para llegar "tan cerca como sea posible de una distribución igual de la salud" no pueden dejar de carecer de cualidades que tendemos a asociar con el proceso de la equidad en salud.

Tengo que dejar claro que no estoy defendiendo que se le dé prioridad a la equidad de proceso sobre todas las demás consideraciones, entre ellas la equidad en salud y la posibilidad de lograr buena salud. Culyer y Wagstaff aciertan cuando se resisten a ello y no hubieran acertado más si en general le hubieran dado una prioridad absoluta a la equidad en la prestación de servicios de salud, independientemente de sus consecuencias. No nos llevan de la sartén al fuego, sino del fuego a la sartén. Pero yo no quiero estar en el fuego ni en la sartén. La equidad en salud es una disciplina amplia e inclusiva, de modo que todo criterio unifocal como el "turno equitativo" o "la distribución igualitaria de la salud" no pueden dejar de excluir muchos otros aspectos importantes. Las características asertivas de los puntos de vista de Williams, Culyer, Wagstaff y otros merecen ser reconocidas y apoyadas, pero eso no implica la negación de la importancia de otros aspectos, como parecen querer ellos cuando les dan una prioridad incondicional a sus criterios unifocales.

Para concluir, la equidad en salud tiene muchos aspectos y lo mejor es verla como un concepto muldimensional. Incluye aspectos relacionados con el logro de la salud y la posibilidad de lograr buena salud, y no solo con la distribución de la atención sanitaria. Pero también incluye la justicia de los procesos $\mathrm{y}$, por lo tanto, debe prestar atención a la ausencia de discriminación en la prestación de la asistencia sanitaria. Además, un buen compromiso con la equidad en salud también necesita que las consideraciones sobre la salud se integren en los temas más amplios de la justicia social y de la equidad global, prestando suficiente atención a la versatilidad de los recursos y a las diferencias de alcance e impacto de los diferentes acuerdos sociales.

En este amplio campo de la equidad en salud, claro que es posible proponer criterios particulares que le den más importancia a unos asuntos que a otros. No estoy tratando de proponer aquí una fórmula que sea exactamente correcta y superior a todas las fórmulas que se puedan proponer, aunque supongo que sería magnífico ser capaz de dar una respuesta canónica a este complejo tema. Mi objetivo ha sido, más bien, identificar diferentes consideraciones que son relevantes para la equidad en salud y presentar argumentos en contra de cualquier reducción arbitraria del dominio de este concepto inmensamente rico. La equidad en salud es una disciplina amplia y el reconocimiento de este hecho básico debe preceder a la aceptación cualificada de cualquier criterio estrecho destinado a fines específicos y funcionales. Las fórmulas especiales tienen su utilidad, pero no por ello podemos prescindir del marco general e inclusivo. Necesitamos ambos.

Agradecimientos. A Sudhir Anand, Lincoln Chen, Anthony Culyer y Angus Deaton, por sus útiles comentarios. 


\section{REFERENCIAS}

1. Sen A. Inequality reexamined. Cambridge, MA: Harvard University Press, y Oxford: Clarendon Press; 1992.

2. Sen A. Equality of what? En: McMurrin S, ed. Tanner lectures on human values. Cambridge: Cambridge University Press, y Salt Lake City: University of Utah; 1980.

3. Sen A. Collective choice and social welfare. San Francisco: Holden-Day; 1970, y Amsterdam: North-Holland; 1979.

4. Sen A. Maximization and the act of choice. Econometrica 1997;65:745-779.

5. Marmot MG, Shipley MJ, Rose G. Inequalities in death-specific explanations of a general pattern. Lancet 1984;1(8384)1003-1006.

6. Marmot MG, Smith GD, Stansfeld S, Patel C, North F, Head J, White I, Brunner E, Feeney A. Health inequalities among british civil servants: The Whitehall II Study. Lancet 1991; 337:1387-1393.

7. Marmot MG, Bobak M, Smith GD. Explorations for social inequalities in health. En Amick BC, Levine S, Tarlov AR, Chapman D, eds. Society and Health. London: Oxford University Press; 1995.

8. Wilkinson RG. The unhealthy societies: the afflictions of inequality. New York: Routledge; 1996.

9. Sen A. Well-being, agency and freedom: the Dewey lectures 1984. Journal of Philosophy 1985;82:169-221.
10. Sen A. Consequential evaluation and practical reason. Journal of Philosophy 2000;97: 477-502.

11. Rawls J. A theory of justice. Cambridge, MA: Harvard University Press; 1971.

12. Murray CJL. Quantifying the burden of disease: the technical basis for disability adjusted life years. Bull World Health Organ 1994;72: 429-445.

13. Murray CJL, López AD. The global burden of disease. Cambridge, MA: Harvard University Press; 1996.

14. World Health Organization. World health report 2000. Geneva: WHO; 2000.

15. Anand S, Hansen K. Disability adjusted life years: a critical review. J Health Econ 1997; 16:685-702.

16. Anand S, Hansen K. DALYs: efficiency versus equity. World Development 1998;26: 307-310.

17. Williams A. If we are going to get a fair innings, someone will need to keep the score! En Barer ML, Getzen TE, Stoddart GL, eds. Health, health care and health economics. New York: Wiley; 1998. p. 330.

18. Culyer AJ, Wagstaff A. Equity and equality in health and health care. J Health Econ 1993;12:431-457.

19. Culyer AJ. Equality of what in health policy? Conflicts between contenders. York
Centre for Health Economics, University of York; 1995. (Discussion Paper No. 142).

20. Mooney G. Economics, communitarianism, and health care. En Barer ML, Getzen TE, Stoddart GL, eds. Health, health care and health economics. New York: Wiley; 1998.

21. Schneider-Bunner C. Equity in managed competition. En Barer ML, Getzen TE, Stoddart GL, eds. Health, health care and health economics. New York: Wiley; 1998.

22. Bleichrodt $\mathrm{H}$. Health utility indices and equity considerations. En Barer ML, Getzen TE, Stoddart GL, eds. Health, health care and health economics. New York: Wiley; 1998.

23. Hurley J. Welfarism, extra-welfarism and evaluative economic analysis in the health sector. En Barer ML, Getzen TE, Stoddart GL, eds. Health, health care and health economics. New York: Wiley; 1998.

24. Rice T. The desirability of market-based health reforms: a reconsideration of economic theory. En Barer ML, Getzen TE, Stoddart GL, eds. Health, health care and health economics. New York: Wiley; 1998.

25. Williams A. Intergenerational equity: an exploration of the 'fair innings' argument. Health Econ 1997:6:117-132.

\section{Congreso Regional de Información en Ciencias de la Salud}

Fechas: 7 a 9 de mayo de 2003

Lugar: Centro de Convenciones de Puebla, México

Tema: Información para las decisiones en salud

日 Instituto Nacional de Salud Pública de México y el Sistema Latinoamericano y del Caribe de Información en Giencias de la Salud (BIREME) le extienden una cordial invitación para participar en el $6^{\circ}$ Congreso Regional de Información en Ciencias de la Salud (CRICS 6).

La serie CRICS se inició en 1992 con ocasión de la III Reunión Bianual del Sistema Latinoamericano y del Caribe de Información en Ciencias de la Salud. Su objetivo principal es proporcionar un foro abierto de discusión de temas importantes relacionados con la información y el uso y aplicaciones de las nuevas tecnologías de la información en el campo de las ciencias de la salud, todo ello con un fuerte componente educacional. La serie CRICS está estructurada en tres días de actividades compuestas por una conferencia magistral, mesas redondas, paneles, conferencias y cursos. 日 CRICS constituye hoy día uno de los acontecimientos importantes en este campo, que en su última convocatoria reunió a cerca de 600 participantes de diferentes países de América Latina y el Caribe, Estados Unidos y Europa. Los congresos son promovidos por la Organización Panamericana de la Salud/ Organización Mundial de la Salud a través de BIREME, que también coordina el Comité Científico.

En esta ocasión el tema central será "Conocer para decidir: la información científico técnica para decisiones en salud". La Conferencia Magistral versará sobre "Información, culturay sociedad". Los seis paneles abordarán los siguientes temas: "Participación social en la sociedad de la información", "Políticas públicas en información científica", "La comunicación científica en la toma de decisiones", "Comunicación, medios y comunidades - el local y el global", "Oreando capacidades para la toma de decisiones en salud" y "Sistemas de información para la toma de decisiones".

\section{Información:}

Teléfono: (52) 22254855293

Correo electrónico: crics@insp.mx

Internet: http://www.crics.info 